\title{
Anmerkungen zur Indikation
}

\author{
G. Hierholzer
}

\section{Zusammenfassung}

Die Indikation zur Korrekturosteotomie bei posttraumatischen Achsenabweichungen ist ein exemplarisches Beispiel für die Komplexität der zu treffenden chirurgisch-operativen Entscheidungen. So unverzichtbar die naturwissenschaftlichen Grundlagen der operativen Therapie sind, so müssen bei der Indikation auch biologische Faktoren, subjektive Beschwerden und die Einstellung des Patienten berücksichtigt werden. Im Einzelfall haben die besprochenen Faktoren eine die Indikation unterschiedlich beeinflussende Bedeutung.

\section{Einleitung $[5,9]$}

Die Ableitung zur Osteotomie und Korrekturosteosynthese nach in Achsenabweichung verheilten Frakturen ergibt sich aus der Analyse verschiedener Faktoren. Diese sind zwar im einzelnen zu ermitteln, dann aber vergleichend $\mathrm{zu}$ bewerten, um zu einer sinnvollen und bei jedem Patienten neu zu treffenden Entscheidung zu kommen. Die Besprechung der speziellen Fragen der Indikaktionsstellung setzt die Prüfung und Beachtung des Allgemeinzustandes des Patienten voraus. Die speziellen Gesichtspunkte der Indikation können wie folgt gegliedert werden [5].

1. die pathomechanische Belastung der Gelenke

2. der funktionelle Gesichtspunkt

3. Auswirkungen auf den Kapselbandapparat angrenzender Gelenke

OP-JOURNAL 2000; 16: 4-6

(c) Georg Thieme Verlag Stuttgart · New York
4. der morphologische Zustand des Knochens, des Knorpels und der Weichteile

5. subjektive Beschwerden

6. kosmetische Auswirkungen

Die Indikation zur Korrekturosteotomie leitet sich aus der vergleichenden Analyse verschiedener Faktoren $a b$

\section{Pathomechanische Gelenkbelastung $[1,2,6]$}

Eine posttraumatische Achsenabweichung hat an der oberen Extremität überwiegend eine funktionelle Bedeutung. An der unteren Extremität kann darüber hinaus die pathomechanische Belastung der angrenzenden Gelenke zum vorrangigen Gesichtspunkt werden. Die Erklärung besteht darin, dass sich an der unteren Extremität der Vektor der Druckbelastung aus der resultierenden von einwirkender Muskelkraft und Körpergewicht errechnet. Die Druckbeanspruchung der Gelenke der unteren Extremität ist demgegenüber derjenigen der oberen Gliedmaße also größer. Die resultierende Druckkraft im Hüftgelenk kann bis zur 4,5-fachen Größe des Körpergewichts ansteigen. Der auf den Hüftkopf und Schenkelhals einwirkende Kraftvektor zeigt die Dislokationstendenz bei Frakturen in diesem Bereich und weist u.a. auf die Gefahr der Varusabweichung hin. Das Behandlungsprinzip einer in Varusposition abgeheilten Fraktur besteht in der valgisierenden Osteotomie mit Wiederherstellung des physiologischen Schenkelhals-Femurschaftwinkels, mit der die pathomechanische Belastung und die muskuläre Insuffizienz beseitigt werden kann. Das Behandlungsziel ist technisch standardisiert und mit großer Erfolgsaussicht verbunden.

Die entgegengesetzte Achsenabweichung, die posttraumatische Coxa valga mit einer unphysiologischen Druckerhöhung und Druckkonzentration im lateralen Gelenkbereich, kann dagegen das Er- gebnis einer Therapie sein, die eingangs durchaus berechtigt war. Bei der Behandlung einer Schenkelhalsfraktur mit steilem Bruchwinkel zur Horizontalen ist der Erhaltung oder der Wiederherstellung der Vitalität des Hüftkopfes Vorrang einzuräumen. Bei steilem Schenkelhalsbruchwinkel kann die Umwandlung der dislozierenden Kräfte in eine interfragmentäre Kompression nur mit der valgisierenden Operationstechnik erzielt werden. Es ist festzustellen, dass in den letzten Jahren diese Indikation zur primär valgisierenden intertrochantären Osteotomie allgemein Anwendung findet, Langzeituntersuchungen über die Folgeerscheinungen jedoch nicht vorliegen. Die therapeutisch verursachte Änderung der Druckbeanspruchung im Schenkelhalsbereich und damit im angrenzenden Hüftgelenk kann Anlass geben für eine sekundäre Varisationsosteotomie, sobald sich an Hüft- und Kniegelenk subjektive Beschwerden oder degenerative Veränderungen einstellen.

Eine pathomechanische Belastung führt an den einzelnen Gelenken zu unterschiedlichen Folgen

Am Unterschenkel werden Achsenabweichungen nach Frakturen insbesondere in der Frontal- und Sagittalebene zunehmend beachtet. Klinische Beobachtungen zeigen, dass die sich aus einer Antekurvationsabweichung des Femurs für das Kniegelenk ergebende mechanische Konsequenz oft unterschätzt wird. Da im Kniebereich an der Vorderseite gelenküberbrückend kräftige Muskelstrukturen zur Verfügung stehen, ist bei einer Antekurvationsabweichung die Stabilität des Gelenkes durch Erhöhung der einwirkenden Muskelkraft gewährleistet. Sie geht mit einer Druckerhöhung im Kniegelenk einher, die überwiegend den Femoropatellaranteil betrifft. Die funktionelle Kompensation der Achsenabweichung mit der andauernd erhöhten Druckbeanspruchung verursacht im weiteren Verlauf eine posttraumatische Arthrose am Knie- 
gelenk und insbesondere am Femoropatellargelenk. Am Unterschenkel führt die Varusabweichung von mehr als $5^{\circ}$ zu einer korrekturbedürftigen Druckkonzentration des Kniegelenks und des oberen Sprunggelenkes. Hier wird also die pathomechanische Druckverteilung zum bestimmenden Faktor für die Indikation zur korrigierenden Osteotomie.

Eine funktionelle Kompensation nach einer Achsenabweichung schützt nicht vor einer posttraumatischen Arthrose

\section{Der funktionelle Gesichtspunkt [5]}

Die klinische Aufgabe, die funktionelle Auswirkung einer posttraumatischen Achsenabweichung richtig zu beurteilen, ist verantwortungsvoll und nicht immer leicht zu lösen. Mit dem korrigierenden Eingriff soll die Gelenkbeweglichkeit verbessert oder erhalten werden. Andererseits kann aber die funktionelle Auswirkung der mit Sekundärschädigungen einhergehenden Achsenabweichung Anlass geben, im therapeutischen Konzept auf die Beweglichkeit eines Gelenkes teilweise oder ganz zu verzichten. Es wird in diesem Falle der noch höherwertige funktionelle Gesichtspunkt wie z.B. die Gehfähigkeit vorangestellt.

Rotationsbewegungen am Femur treten häufiger auf, als sie offensichtlich diagnostiziert werden. Es handelt sich um eine Achsenabweichung, die ohne Veränderung der Druckbelastung des kugelartigen Hüftgelenkes einhergeht. Eine pathomechanische Beanspruchung des angrenzenden Kniegelenkes kann für längere Zeit untergeordnet bleiben. Besteht eine Außenrotationsabweichung, so kann diese teilweise oder ganz muskulär kompensiert werden. Die fortwährende Überbeanspruchung der innenrotierenden und adduzierenden Muskeln verursacht im weiteren Verlauf eine funktionelle Störung beim Gehen, die neben subjektiven Beschwerden zum bestimmenden Faktor für eine Korrekturosteotomie wird. Auch bei einer in Varusstellung abgeheilten hüftgelenknahen Fraktur mit verändertem Hebelarm kann die muskuläre Insuffizienz mit positivem Trendelenburgschen Zeichen $\mathrm{zu}$ einer im Vordergrund stehenden funktionellen Störung führen. Als Beispiel am Unterschenkel stört eine Rotations- und Varusabweichung den Abrollvorgang des Fußes, verursacht Gehunsicherheit und kann damit gegenüber den anderen Faktoren zum vorrangigen Gesichtspunkt werden.
Die funktionelle Auswirkung einer zu stark ausgeprägten Beugestellung nach einer Operation zur Arthrodese des Kniegelenkes ist sehr eindrücklich. Eine Flexionsstellung von mehr als $15^{\circ}$ verursacht teilweise oder weitgehend eine Behinderung der Gehfähigkeit. Diese schwerwiegende funktionelle Störung ist durch eine extendierende Osteotomie mit dem Eingriff zur Rearthrodese zu beseitigen.

Die traumatische Hüftluxation mit nachfolgender Coxarthrose und einer in Achsenabweichung verheilten Femurfraktur verursachen Instabilität und schmerzhafte Bewegungseinschränkung. Der Verlust der Gehfähigkeit hat klinisch Vorrang vor weiteren funktionellen Überlegungen. Die hauptsächliche Aufgabe der korrigierenden Osteotomie besteht in der Wiederherstellung der Gehfähigkeit. Die komplexe Problematik führt schließlich doch zu einer klaren Indikation.

Für die Indikation zur Korrekturosteotomie kann der funktionelle Gesichtspunkt eine sehr unterschiedliche Bedeutung haben.

\section{Auswirkungen auf den Kapselbandapparat [5,7]}

Art und Ausmaß knöcherner Achsenabweichungen nach Frakturen können Auswirkungen auf die statisch und dynamisch stabilisierenden Weichteilstrukturen haben. Bei Varus- und Valgusabweichungen werden diese auf der Konvexseite einer unphysiologischen Zugbeanspruchung unterzogen und unterliegen damit einer Dehnung und Lockerung. Auf der Konkavseite kann eine Atrophie und Schrumpfung von Strukturen eintreten, und es besteht die Gefahr eines Circulus vitiosus. Bei einer Antekurvationsstellung ergibt sich aus den gelenküberbrückenden dynamisch stabilisierenden Strukturen eine gewisse Kompensationsfähigkeit. Am Kniegelenk reichen aber die dorsal stabilisierenden Strukturen nicht aus, um bei einer Rekurvationsstellung das Gleichgewicht des Gelenkes zu gewährleisten. In diesem Beispiel münden die Achsenabweichung der Gelenkflächen und die Überdehnung der Kapselbandstrukturen in einen Circulus vitiosus ein.

Posttraumatische Deformierungen am Fuß sind häufig die Ursache eines schmerzhaften Reizzustandes, der dann zusätzlich zur Weichteilkontraktur führt. Das klinische Erscheinungsbild mit der gestörten Funktion und mit den subjektiven Beschwerden muss dann hinsichtlich beider Ursachenfaktoren analysiert werden. Das Ausmaß der Veränderungen am Kapselbandapparat und insbesondere der eingetretenen Schrumpfung der Strukturen wird u.U. erst bei der operativen Revision ersichtlich und erfordern dann eine Ausdehnung des operativen Eingriffes im Sinne der Arthrolyse.

Die Abheilung einer Fraktur in Achsenabweichung mit Zusammensinterung von Knochengewebe im interligamentären Kniebereich kann schließlich zu dem Phänomen der relativen Bandinsuffizienz führen. Am Kniegelenk ist dies nicht selten zu beobachten. Die Auswirkung der Achsenabweichung besteht u.a. in einer klinisch nachweisbaren relativen Instabilität des Kapselbandapparates, die durch eine aufrichtende Osteotomie mit Wiederherstellung der knöchernen Distanz beseitigt wird.

Die Auswirkungen einer Achsenabweichung können am Kapselbandapparat zu Schäden führen, die in einen Circulus vitiosus einmünden.

\section{Bedeutung einer morphologischen Schädigung $[1,5]$}

Die in Verbindung mit dem Trauma oder in der Folgezeit aufgetretene Schädigung der Knorpel- und Knochenstrukturen und der umgebenden Weichteile können ebenfalls die Indikation für eine korrigierende Osteotomie und die anzuwendende Operationstechnik beeinflussen. So nimmt z. B. die Erfolgsaussicht für die Bedeutung einer knöchernen Achsenabweichung mit dem Ausmaß einer bereits eingetretenen Knorpelschädigung der angrenzenden Gelenke ab. Ist der arthrotische Prozess fortgeschritten, so kann sich hieraus die Kontraindikation für eine gelenkerhaltende Osteotomie ergeben. Die Korrektur erfolgt u.U. in Verbindung mit dem Eingriff zur Arthrodese. Sind aber bei einer Achsenabweichung im angrenzenden Gelenk Knorpelbereiche noch erhalten, so wird die Planung zur korrigierenden Osteotomie diese als Belastungsflächen nutzen, teilweise unter Inkaufnahme einer gewissen unphysiologischen Position. Als Beispiel verweisen wir auf die röntgenologisch deutliche Erweiterung des Kniegelenkspaltes nach einer varisierenden Osteotomie mit leichter Überkorrektur, mit der die Funktion erhalten und ein Rückgang der Beschwerden erreicht werden kann. 
Ein posttraumatischer Teilschaden eines Gelenkes kann zur Indikation einer Osteotomie „im Sinne der Überkorrektur" führen

Eine Veränderung des Knochengewebes nach vorangegangener Infektion oder z. B. in Form der Sklerose aus anderer Ursache beeinflusst die Lokalisation einer Korrekturosteotomie. Ist die Veränderung stark ausgeprägt, so wird die Korrektur nicht im Maximum der Achsenabweichung durchgeführt. Ergibt sich bei bestehender Infektion eine Indikation zur Osteotomie, so erfolgt diese nach Abklingen der Entzündung und entsprechend den dafür gültigen Richtlinien. Die Gewebeschädigung nach einer Infektion an Knochen- und Weichteilgewebe beeinflusst auch die Wahl der Korrekturosteosynthesetechnik. Für diese Bedingungen ist die überbrückende Fixateur-externeOsteosynthese $\mathrm{zu}$ empfehlen, mit der eine Implantateinbringung in den gefährdeten Bereich vermieden wird. Die Schädigung der Haut und der darunter gelegenen Weichteilschichten in Form ausgedehnter Narbenbildungen oder z. B. bei einer fortgeschrittenen arteriellen Durchblutungsstörung erhöhen das Infektionsrisiko und sind bei der Wahl der operativen Zugänge zu berücksichtigen.

\section{Bedeutung subjektiver Beschwerden $[2,3,9]$}

Die Art und das Ausmaß der subjektiven Beschwerden, die in Verbindung mit einer posttraumatischen Achsenabweichung auftreten, können für die Indikationsstellung zur operativen Korrektur ebenfalls eine vorrangige Bedeutung gewinnen.

Der präventive Gesichtspunkt wiegt u.U. schwerer als ein fehlendes oder geringes Beschwerdebild

Aus der Elastizität des Gelenkknorpels und aus einer trainierten Muskulatur ergibt sich in diesem Alter für viele Achsenabweichungen eine Kompensationsfähigkeit, die subjektive Beschwerden u.U. erst verzögert auftreten lassen. Im jugendlichen Alter ergibt sich aus fehlenden oder gering ausgebildeten subjektiven Beschwerden aus diesen Erkenntnissen keine Kontraindikation für die korrigierende Osteotomie, sofern diese aus gelenkmechanischer Gesetzmäßigkeit angezeigt ist. Als Beispiel kann auf eine erhebliche Varusabweichung am distalen
Unterschenkel hingewiesen werden, die zum Zeitpunkt des korrigierenden Eingriffes beim jugendlichen Alter noch keine wesentlichen subjektiven Beschwerden verursachte. Andererseits kann im höheren Lebensalter ein mäßiges Beschwerdebild Anlass geben, von einer röntgenologisch angezeigt erscheinenden Osteotomie abzusehen. Bei einer verhältnismäßig geringen Lebenserwartung tritt dann der Gesichtspunkt einer prophylaktischen Korrekturosteotomie zurück.

Zu beachten ist weiterhin die Art der subjektiven Beschwerden, die ihrerseits zum tragenden Gesichtspunkt werden kann. So ist z. B. bei einer Patientin im hohen Lebensalter mit einer Varusabweichung nach distaler Femurfraktur die geklagte Gehunsicherheit vorrangig gegenüber der pathomechanischen Druckbeanspruchung des angrenzenden Gelenkes und gegenüber dem mäßigen Beschwerdebild. Andererseits wird an diesem Beipiel wiederum die Überschneidung der Faktoren „funktionelle Auswirkungen und subjektive Beschwerden“ deutlich.

\section{Kosmetische Auswirkungen [5,8]}

Die Einbeziehung dieser Frage in die Indikation zur korrigierenden Osteotomie nach posttraumatischen Achsenabweichungen ist durchaus berechtigt. Wir verstehen darunter allerdings nur diejenigen kosmetischen Auswirkungen, deren Korrekturbedürftigkeit einer kritischen ärztlichen Einstellung gerecht wird. Dafür seien 2 typische Beispiele benannt. Eine erhebliche posttraumatische Verkürzung der unteren Extremität kann, abgesehen von der zusätzlichen funktionellen Bedeutung, besonders beim jungen Menschen zu schwerwiegenden psychischen Problemen führen. Natürlich muss die Indikation zur Verlängerungsosteotomie der Nutzen-Risiko-Analyse gerecht werden. Die Bedeutung der kosmetischen Auswirkungen einer posttraumatischen Achsenabweichung ist auch an dem Beispiel eines Patienten am Ende des Wachstumsalters ersichtlich, bei dem aufgrund ausgedehnter Verbrennungen eine Schienbeinkopffraktur in der Phase der Erstbehandlung nicht zufriedenstellend stabilisiert werden konnte.

Die Beurteilung der kosmetischen Auswirkung einer Achsenabweichung ist ärztlicherseits besonders verantwortungsvoll

\section{Literatur}

1 Bombelli R. Structure and function in normal and abnormal hips. Springer, Berlin, Heidelberg, New York, Tokyo 1993

2 Denoth J. Analyse von Belastung und Beanspruchung des Bewegungsapparates. Swiss Med 1987; 9: 35-41

3 Franzreb M, Strecker W, Kinzl L. Wertigkeit der klinischen Untersuchung von Torsionswinkel- und Längenverhältnissen der unteren Extremität. Akt Traumatol 1995; 25 : $153-156$

${ }^{4}$ Gürke L, Martinoli S. Korrektur mehrdimensionaler Deformitäten durch eine einzige Osteotomie. In: Strecker W, Keppler P. Kinz L. Posttraumatische Beindeformitäten. Springer, Berlin, Heidelberg, New York, Tokyo 1997

5 Hierholzer G, Müller K.-H. Korrekturosteotomien nach Traumen an der unteren Extremität, Springer, Berlin, Heidelberg, New York, Tokyo 1984

${ }^{6}$ Maquet GJ. Biomechanic of the knee Springer, Berlin, Heidelberg, New York, Tokyo 1984

7 Morscher E, Hefti F, Baumann JU. Kombinierte laterale Bandplastik und Calcaneusosteotomie bei der recidivierenden Distorsio pedis. Orthopädee 1986; 15: 461 -465

8 Pfeil J, Grill F, Graf R. Extremitätenverlängerungen, Deformitätenkorrektur, Pseudarthrosenbehandlung Springer, Berlin, Heidelberg, New York, Tokyo 1996

9 Strecker W, Keppler P, Kinzl L. Posttraumatische Beindeformitäten. Springer, Berlin, Heidelberg, New York, Tokyo 1997

Prof. Dr. G. Hierholzer

Radolfzeller Straße 109

78478 Allensbach 